\title{
Manual discrimination of force using active finger motion
}

\author{
X. D. PANG, H. Z. TAN, and N. I. DURLACH \\ Massachusetts Institute of Technology, Cambridge, Massachusetts
}

\begin{abstract}
In these experiments, two plates were grasped between the thumb and forefinge: and squeezed together along a linear track. An electromechanical system presented a constant resistance force during the squeeze up to a predetermined location on the track, whereupon the force effectively went to infinity (simulating a wall) or to zero (simulating a cliff). The task of the subject was to discriminate between two alternative levels of the constant resistance force (a reference level and a reference-plus-increment level). Results of these experiments indicate a just noticeable difference of roughly $7 \%$ of the reference force using a one-interval paradigm with trial-by-trial feedback over the ranges $2.5 \leq F_{0} \leq 10.0$ newtons, $5 \leq D \leq 30 \mathrm{~mm}, 45 \leq S \leq 125 \mathrm{~mm}$, and $25 \leq V \leq$ $160 \mathrm{~mm} / \mathrm{sec}$, where $F_{0}$ is the reference force, $D$ is the distance squeezed, $S$ is the initial fingerspan, and $V$ is the mean velocity of the squeeze. These results, based on tests with 5 subjects, are consistent with a wide range of previous results, some of which are associated with other body surfaces and muscle systems and many of which were obtained with different psychophysical methods.
\end{abstract}

This is the second in a series of papers concerned with the manual perception of objects, and, more specifically, with the ability to distinguish between different objects manually (i.e., with manual resolution). In the first paper, we reported the results of a variety of experiments in which the subject was required to discriminate or identify object length by means of the finger-span method (Durlach et al., 1989). In these experiments, a rigid object was grasped between the terminal pads of the thumb and forefinger, and object length was estimated by sensing the differential position of these pads. In the present series of experiments, an object was again grasped between the terminal pads of the thumb and forefinger; in this case, however, the object was not rigid, and the task was to squeeze the object and estimate the resistance force. The experimental apparatus was designed in such a way that the force was constant over the displacement resulting from the squeeze, the force was varied between squeezes, and the task was to discriminate between two alternative levels of the force. In the length-resolution task, the response is derived from estimates of finger position. In the current task, the response is derived from estimates of finger force. In both cases, perceptual cues

This work was supported by ONR Grants N00014-88-K-0338, N00014 89-J-3247, and NO0014-90-J-1935, NIH Grant 2-R01-DC00126-11, Fairchild foundation funds awarded to X. D. Pang, and a Chu Fellowship to H. Z. Tan. The support and contributions of John Hollerbach at all levels of this work are greatly appreciated. We would also like to thank Bill Rabinowitz for his help with the instrumentation and other aspects of the work. We appreciate the contributions of Younes Borki, Lorraine Delhorne, Mary Hou, and Mandayam Srinivasan. Correspondence should be addressed to N. I. Durlach, Research Laboratory of Electronics and Department of Engineering and Computer Science, Massachusetts Institute of Technology, Cambridge, MA 02139. are available from both the cutaneous sensory system and the kinesthetic/proprioceptive sensory system (see, e.g., the review by Clark \& Horch, 1986). In general, our objective in these studies is to provide basic psychophysical information that can be used both to advance our understanding of manual perception and as a background for improved design of robots, teleoperators, and virtual environment systems.

In the three sections of this paper that follow, we describe our experimental methods, provide the results, and comment on the results and compare them to relevant previous data in the literature. Additional information about the experimental apparatus, about statistical assumptions and data processing, and about learning effects is included in Appendices A-C.

\section{METHOD}

The experimental apparatus had two parallel plates, one of which was fixed and the other of which could be moved along a linear track perpendicular to the plates (see Figure 1). The subject grasped the two plates between the thumb and forefinger (with the thumb on the movable plate) and squeezed the movable plate toward the fixed plate. The control algorithm of the apparatus was designed so that the force resisting the squeeze was constant over the length and duration of the squeeze, and the subject was required to discriminate between two alternative levels of this resistance force. The discrimination capability was measured as a function of the reference or base force $F_{0}$, the initial finger-span $S$ between the plates, the pushing distance $D$ (equal to the initial span minus the terminal span), and the mean pushing velocity $V$. In addition, two methods of terminating the push were compared. In one case (denoted MW), the push was terminated by a mechanical wall (i.e., for all practical purposes, the resistance force increased to infinity at the terminal span). In the other case (denoted EC), the mechanical wall was replaced by an electrical cliff (i.e., the resistive force dropped to approximately zero at the terminal span). The mean push- 


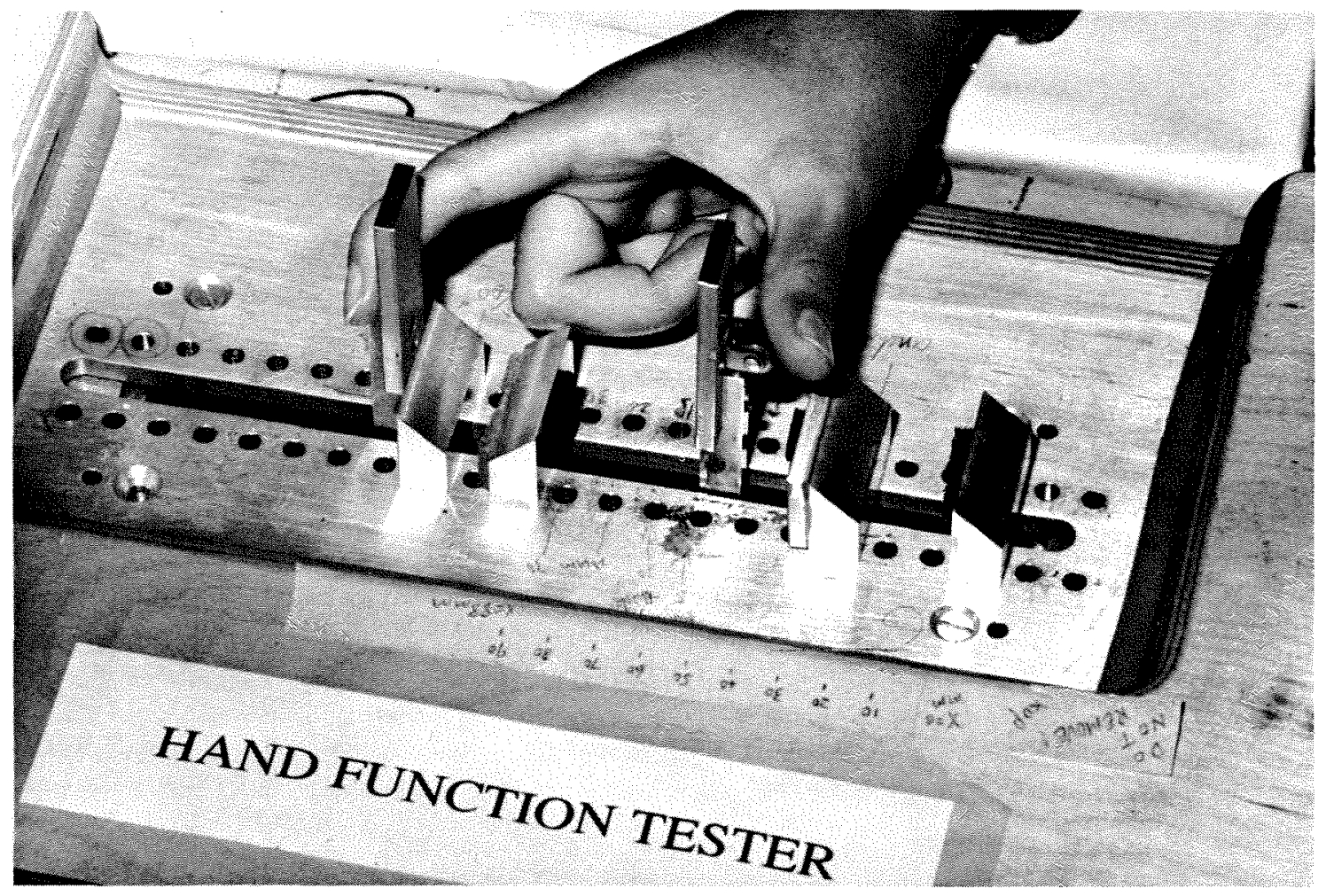

Figure 1. Electromechanical device used to measure manual force resolution.

ing velocity, which was automatically measured on each trial, was controlled (to at least some degree) by means of instructions to the subject and feedback after each push. Except in the runs used explicitly to measure the effect of velocity, instructions were always to push at "the most comfortable speed." Further details on the design and performance of the apparatus are available in Appendix A.

In all cases, subjects were tested using a one-interval, twoalternative, forced-choice paradigm with trial-by-trial correct-answer feedback. The forces to be discriminated, $F_{0}$ and $F_{0}+\Delta F$, were presented with equal a priori probabilities (except when determining the receiver operating characteristic [ROC] curves discussed in Appendix B), and each run consisted of 64 trials with the experimental parameters held fixed. The experimental sequence was under computer control, and each run was typically completed in less than $5 \mathrm{~min}$. Five subjects free of hand disorders (ages 22-45) participated in the experiments, each of whom used the right (dominant) hand for testing, and at least 3 of whom were used to measure resolution for each set of parameter values. The subjects always wore earplugs to eliminate any possible auditory cues to the identity of the stimulus $\left(F_{0}\right.$ or $\left.F_{0}+\Delta F\right)$.

The $2 \times 2$ response matrix obtained from each 64 -trial run was processed to obtain estimates of the sensitivity index $d^{\prime}$ and the response bias $\beta$ (e.g., see Berliner \& Durlach, 1973). In this method of data processing, it is assumed that the underlying density functions associated with the two stimuli being discriminated are normal and of equal variance (means $M_{1}$ and $M_{2}$, and variance $\sigma^{2}$ ); $d^{\prime}$ is defined as the difference between the means divided by the square root of the variance $\left[d^{\prime}=\left(M_{1}-M_{2}\right) / \sigma\right]$, and $\beta$ is defined as the deviation of the response criterion $(C)$ from the midpoint between the two means $\left.\left[M_{1}+M_{2}\right) / 2\right]$ divided by the square root of the variance $\left\{\beta=\left[C-\left(M_{1}+M_{2}\right) / 2\right] / \sigma\right\}$. The condition $\beta=0$ corresponds to unbiased response behavior and, given such behavior the condition $d^{\prime}=1$ corresponds to approximately $75 \%$ correct performance. Generally speaking, the values of $\beta$ in this study were found to be negligibly different from zero (i.e., no significant bias was observed), and the values of $d^{\prime}$ were found (as usual) to be roughly proportional to the increment $\Delta F$, or, for fixed $F_{0}$, to the fraction $\Delta F / F_{0}$ (see Appendix B). Given this proportionality, performance can be summarized by the proportionality constant $\delta^{\prime}=d^{\prime} /\left(\Delta F / F_{0}\right)$ or, taking account of "measurement noise," by the quantity $\bar{\delta}^{\prime}$, which denotes the average of $\delta^{\prime}$ over the different values of $\Delta F$ tested for fixed $F_{0}$. If the just noticeable difference, JND $(\Delta F)_{0}$, is defined by the performance threshold $d^{\prime}=1$, then $\delta^{\prime}=1 /\left[(\Delta F)_{0} / F_{0}\right]$ is the reciprocal of the Weber fraction at the reference force $F_{\mathbf{0}}$. In all cases, the results [denoted force JND (\%)] give the value of $\left(1 / \bar{\delta}^{\prime}\right) \times 100$ (see the Results section). This quantity corresponds to the value of the Weber fraction in percent using the performance threshold $d^{\prime}=1$ (but making use of all the data gathered at $F_{0}$, not just the data near $d^{\prime}=1$ ). If we had plotted $\overline{1 / \delta^{\prime}}$ instead of $1 / \bar{\delta}^{\prime}$, the difference would not have been visually detectable on the graphs shown.

The parameter values tested are shown in Table 1. The standard set of values was used primarily for methodological checks. Thus, these parameter values were used to examine the extent to which (1) the ROC curves for this task were straight lines of unit slope, (2) the variability of the measured $d^{\prime}$ estimates was consistent with the assumption of Bernoulli statistics, and (3) the average values of the $d^{\prime}$ estimates were proportional to the force increment $\Delta F$. Results on these issues are presented in Appendix B. The other four sets of parameter values were used, as indicated, to measure the dependence of the force JND on base force $F_{0}$, initial finger-span $S$, pushing distance $D$ (including the type of termination at the end of the push), and finally, mean pushing velocity $V$. In general, in 
Table 1

Experimental Parameter Settings

\begin{tabular}{|c|c|c|c|c|c|}
\hline Experimental Condition & $F_{0}$ & $\Delta F / F_{0}$ & $S$ & $D$ & MW or EC \\
\hline Standard & 5.0 & $5,10,15,20$ & 105 & 20 & MW \\
\hline Changing base force $F_{0}$ & $2.5,5.0,10.0$ & $5,10,15$ & 105 & 20 & MW \\
\hline Changing initial finger-span $S$ & 5.0 & $5,10,15$ & $45,80,105,125$ & 10 & MW \\
\hline Changing displacement $D$ & 5.0 & $5,10,15$ & 105 & $5,10,20,30$ & MW \& EC \\
\hline Changing pushing speed $V$ & 5.0 & $5,10,15,20$ & 105 & 20 & MW \\
\hline
\end{tabular}

Note $-F_{0}$ is given in Newtons, $\Delta F / F_{0}$ in percent, $S$ and $D$ in millimeters. $\mathrm{MW}=$ mechanical wall. EC $=$ electrical cliff.

a given experimental session (which lasted roughly $2 \mathrm{~h}$ ), all parameters were held constant except $\Delta F$, which was varied randomly over the given set of values from run to run. The order in which the parameters were studied was $F_{0}, S, D$ (with $\mathrm{MW}$ and $\mathrm{EC}$ ), and $V$. In all cases, the data plotted represent the results obtained after performance appeared stationary and stopped improving due to training. Results on training are presented in Appendix C. The total number of trials performed in the standard condition after training (summed over the subjects) was approximately 28,000 . The total number of trials for the nonstandard conditions after training (summed over the conditions as well as the subjects) was approximately 63,000 .

\section{RESULTS}

The results on the dependence of the force JND (\%)that is, the Weber fraction in percent-on the reference force $F_{0}$, the initial finger-span $S$, the pushing distance $D$, the method of pushing termination (MW or EC), and the pushing speed $V$, are shown in Figures 2-5. Roughly speaking, these results are easy to summarize: The force JND (\%) is essentially independent of all the parameter variables tested and lies in the range $5 \%-10 \%$.

The data shown in Figure 2, which represent (on the average) 640 trials per value of $F_{0}$ per subject, indicate

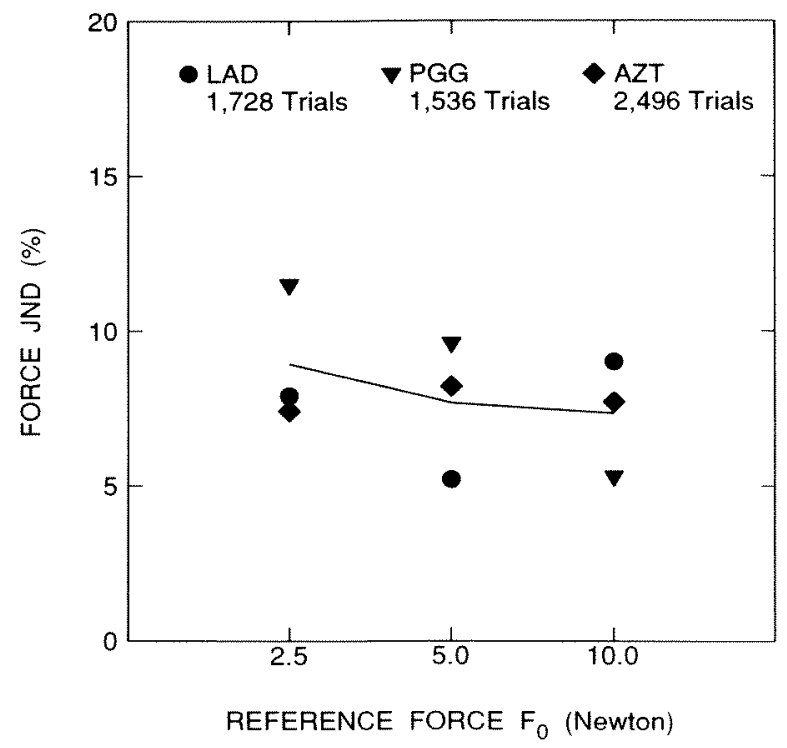

Figure 2. Force JND $(\%)$ versus reference force $F_{0}$. Solid line connects means of data points. Pushing distance $D$ was $20 \mathrm{~mm}$, initial finger-span $S$ was $105 \mathrm{~mm}$, and termination was by mechanical wall (MW). Average JND is 8\%. The horizontal axis is logarithmic. Subiects and number of trials for each subiect are snecified on the granh. that the force JND $(\%)$ is roughly independent of $F_{0}$, and thus the data are consistent with Weber's law over the range $2.5 \leq F_{0} \leq 10.0 \mathrm{~N}$. Although the results for Subject P.G.G. suggest that the force JND (\%) becomes smaller as $F_{0}$ increases over this range, this variation is not supported by the results for Subjects A.Z.T. and L.A.D.

The data shown in Figure 3, which represent (on the average) 725 trials per value of $S$ per subject, indicate that the JND (\%) is roughly independent of $S$ over the range $45 \leq S \leq 125 \mathrm{~mm}$. The exceptionally high point for Subject P.G.G. at $105 \mathrm{~mm}$ is mainly the result of two runs in which $d^{\prime}$ was negative. If one assumes that the subject confused the response code during these runs and reflects these values about zero, the point would be plotted at $8.5 \%$ (or at $7.6 \%$ if the two negative $d^{\prime}$ runs are discarded) rather than at $9.8 \%$.

The data shown in Figure 4A, which represent (on the average) 912 trials per value of $D$ per subject, indicate that the JND (\%) is roughly independent of $D$ over the range $5 \leq D \leq 30 \mathrm{~mm}$. The data shown in Figure 4B, which represent (on the average) 933 trials per value of $D$ per subject, differ from the data shown in Figure 4A in that the tests were performed with EC termination rather than MW termination, and Subject G.Y.O. was sub-

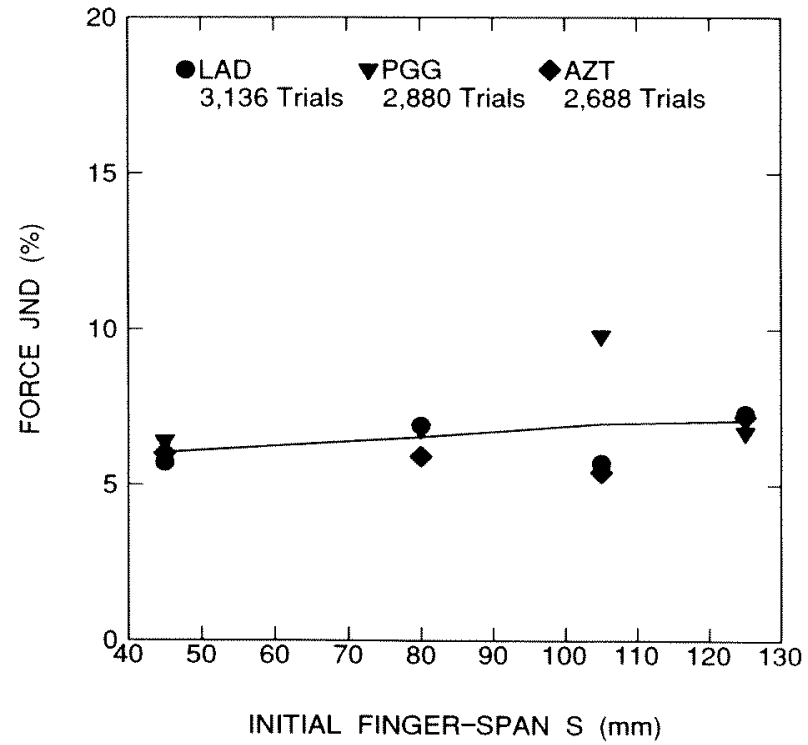

Figure 3. Force JND (\%) versus initial finger-span $S$. Solid line connects means of data points. Reference force $F_{0}$ was $5 \mathrm{~N}$, pushing distance $D$ was $10 \mathrm{~mm}$, and termination was by mechanical wall (MW). Average JND is 7\%. Subjects and number of trials for each subiect are snecified on the oranh. 

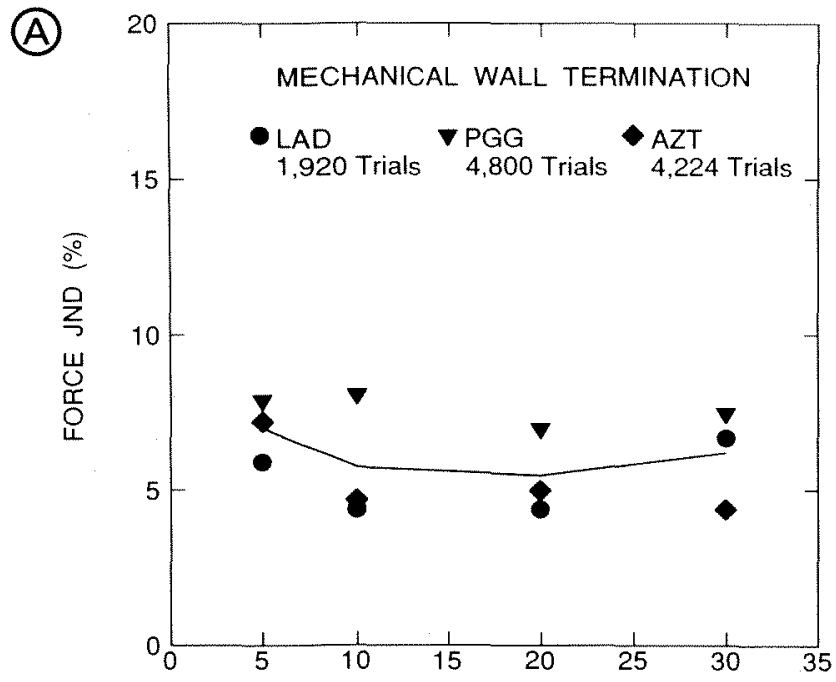

PUSHING DISTANCE D (mm)

(B)

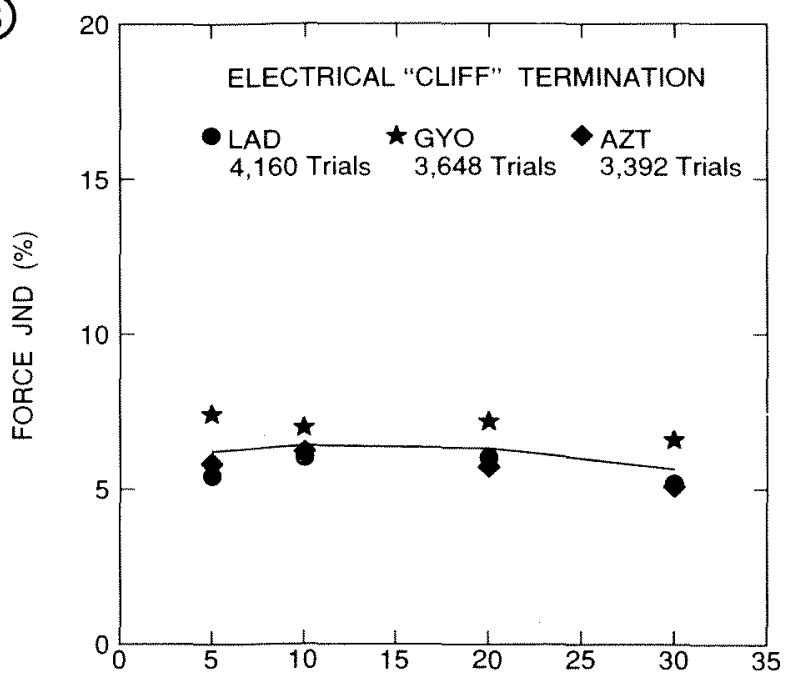

PUSHING DISTANCE D (mm)

Figure 4. Force JND (\%) versus pushing distance $D$. Results for termination by mechanical wall (MW) are shown in Figure $4 \mathrm{~A}$, those for termination by electrical cliff (EC) in Figure 4 B. Solid lines connect means of data points. In both cases, reference force $F_{0}$ was $5 \mathrm{~N}$, initial finger-span $S$ was $105 \mathrm{~mm}$, and average JND is 6\%. Subjects and number of trials for each subject are specified on the graphs. Note that Subject P.G.G. was only tested with MW and that G.Y.O. was only tested with EC (see discussion in text).

stituted for Subject P.G.G. These results again shown that the JND (\%) is roughly independent of $D$. Moreover, since the overall level of the JND (\%) in Figure 4A is approximately the same as it is in Figure 4B, averaging about $6 \%$ in both cases, these data indicate that the JND (\%) is roughly independent of whether the termination is achieved by the MW method or by the EC method. It should be noted, however, that Subject G.Y.O. replaced Subject P.G.G. in this study because P.G.G. could not learn to do the task with EC termination. In other words, even after extensive training, the performance of Subject P.G.G. with EC was much worse than with MW. This is particularly interesting in view of the fact that the results for Subjects L.A.D. and A.Z.T. were essentially the same for the two methods of termination (see, however, the discussion in Appendix $\mathrm{C}$ about learning effects).

The data shown in Figure 5, which represent (on the average) 2,048 trials per value of $V$ per subject, indicate that pushing speed is also not a major factor. For mean pushing velocities in the range $23 \leq V \leq 157 \mathrm{~mm} / \mathrm{sec}$, the JND (\%) remained in the vicinity of $5 \%-10 \%$ of the base force. Only for Subject G.Y.O. did the JND (\%) appear to increase significantly when the velocity was high. For Subject L.A.D., the JND (\%) increased somewhat when the pushing velocity either increased or decreased from the most comfortable speed, and for Subject P.C.N. (a new subject), the JND (\%) was independent of the pushing velocity. Note also that the most comfortable velocity varied with the subject (approximately $60 \mathrm{~mm} / \mathrm{sec}$ for G.Y.O., $90 \mathrm{~mm} / \mathrm{sec}$ for L.A.D., and $153 \mathrm{~mm} / \mathrm{sec}$ for P.C.N.).

Results on the pushing velocities for the data shown in Figures 2-4, all of which were obtained with the subjects instructed to push at the most comfortable speed, are presented in Table 2. For Subject L.A.D. (the only subject who participated in all of the experiments), these results are reasonably consistent with those shown in Figure 5. Except for the changing $S$ tests with $S=45 \mathrm{~mm}$ and the changing $D$ tests with $D=5 \mathrm{~mm}$ and $D=10 \mathrm{~mm}$, all the values of $V$ in Table 2 (including those that correspond directly to the case for which data are plotted in Fig-

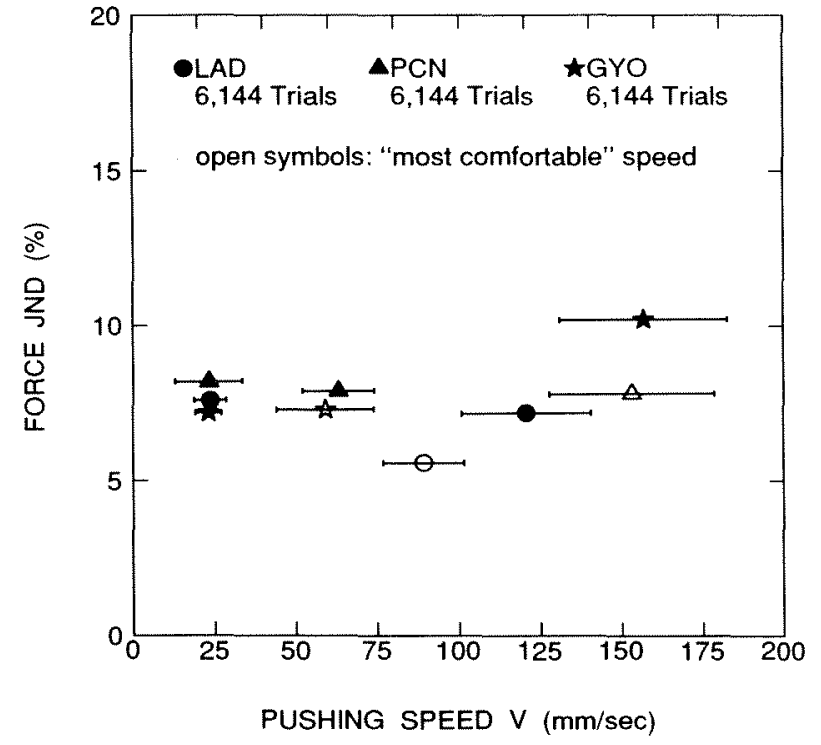

Figure 5. Force JND (\%) versus pushing speed $V$. Graph show $M_{ \pm 1} S D$ for $V$. Reference force $F_{0}$ was $5 \mathrm{~N}$, pushing distance $D$ wa $20 \mathrm{~mm}$, initial finger-span $S$ was $105 \mathrm{~mm}$, and termination was b. mechanical wall (MW). Subject's "most comfortable" pushing speed indicated by open symbols, was $90 \mathrm{~mm} / \mathrm{sec}$ for L.A.D., $153 \mathrm{~mm} / \mathrm{se}$ for P.C.N., and $59 \mathrm{~mm} / \mathrm{sec}$ for G.Y.O. Average JND is $8 \%$. Sub jects and number of trials for each subject are specified on the graph 
Table 2

Means and Standard Deviations of Pushing Speed $V$ (millimeter/second) for Data Presented in Figures 2-4

\begin{tabular}{lcrrrr}
\hline & & \multicolumn{3}{c}{ Subject } & Average \\
\cline { 3 - 5 } Condition & Parameter & L.A.D. & \multicolumn{1}{c}{ P.G.G. } & A.Z.T. & of Means \\
\hline Standard & $\Delta F / F_{0}=5 \%$ & $97.5 \pm 22.3$ & $117.3 \pm 36.6$ & $67.1 \pm 24.7$ & 94.0 \\
& $\Delta F / F_{0}=10 \%$ & $100.0 \pm 16.3$ & $115.5 \pm 30.5$ & $75.5 \pm 27.1$ & 97.0 \\
& $\Delta F / F_{0}=15 \%$ & $98.2 \pm 29.3$ & $116.0 \pm 33.6$ & $74.9 \pm 25.9$ & 96.4 \\
& $\Delta F / F_{0}=20 \%$ & $100.1 \pm 14.7$ & $126.9 \pm 40.8$ & $74.5 \pm 21.1$ & 100.5 \\
Changing $F_{0}$ & $F_{0}=2.5 \mathrm{~N}$ & $100.5 \pm 21.1$ & $22.5 \pm 17.7$ & $47.9 \pm 47.8$ & 57.0 \\
& $F_{0}=5.0 \mathrm{~N}$ & $96.2 \pm 16.4$ & $107.9 \pm 19.3$ & $91.0 \pm 35.7$ & 98.4 \\
& $F_{0}=10.0 \mathrm{~N}$ & $103.6 \pm 17.2$ & $90.0 \pm 21.2$ & $117.5 \pm 49.3$ & 103.7 \\
Changing $S$ & $S=45 \mathrm{~mm}$ & $46.8 \pm 20.0$ & $40.0 \pm 11.6$ & $102.0 \pm 24.6$ & 62.9 \\
& $S=80 \mathrm{~mm}$ & $72.5 \pm 25.1$ & $37.1 \pm 7.1$ & $97.2 \pm 31.9$ & 68.9 \\
& $S=105 \mathrm{~mm}$ & $81.6 \pm 14.0$ & $45.1 \pm 12.9$ & $48.5 \pm 8.2$ & 58.4 \\
Changing $D(\mathrm{MW})$ & $S=125 \mathrm{~mm}$ & $81.5 \pm 12.5$ & $46.4 \pm 10.5$ & $90.5 \pm 17.7$ & 72.8 \\
& $D=5 \mathrm{~mm}$ & $65.7 \pm 24.5$ & $56.9 \pm 16.3$ & $81.4 \pm 22.3$ & 68.0 \\
& $D=10 \mathrm{~mm}$ & $59.1 \pm 9.3$ & $68.0 \pm 15.8$ & $86.1 \pm 32.6$ & 71.1 \\
& $D=20 \mathrm{~mm}$ & $71.5 \pm 14.1$ & $90.1 \pm 18.8$ & $116.9 \pm 29.3$ & 92.8 \\
& $D=30 \mathrm{~mm}$ & $73.8 \pm 15.0$ & $120.5 \pm 23.8$ & $134.9 \pm 35.6$ & 109.7 \\
& & & & \\
Changing $D(\mathrm{EC})$ & $D=5 \mathrm{~mm}$ & $99.0 \pm 56.5$ & $93.5 \pm 48.6$ & $149.7 \pm 53.2$ & 114.1 \\
& $D=10 \mathrm{~mm}$ & $84.9 \pm 41.6$ & $68.5 \pm 36.6$ & $135.4 \pm 62.7$ & 96.3 \\
& $D=20 \mathrm{~mm}$ & $96.7 \pm 73.9$ & $79.4 \pm 36.5$ & $145.2 \pm 28.0$ & 107.1 \\
& $D=30 \mathrm{~mm}$ & $95.4 \pm 101.2$ & $78.4 \pm 12.9$ & $179.2 \pm 30.5$ & 117.7 \\
\hline
\end{tabular}

Note $-\mathrm{MW}=$ mechanical wall. $\mathrm{EC}=$ electrical cliff

ure 5) lie in the same range as that indicated for the most comfortable $V$ in Figure 5 (i.e., roughly $75-100 \mathrm{~mm} / \mathrm{sec}$ ). For Subject G.Y.O., the results on most comfortable $V$ in Table 2 and Figure 5 are less consistent; whereas the results in Table 2 indicate a most comfortable $V$ of roughly $70-90 \mathrm{~mm} / \mathrm{sec}$, the results in Figure 5 suggest a value of $60 \mathrm{~mm} / \mathrm{sec}$. However, the method of termination is different in the two cases. One very puzzling aspect of the data shown in Table 2 concerns the results for changing $D$ : Whereas the most comfortable $V$ tends to increase with $D$ in the MW case, it does not in the EC case. We also have not been able to find a satisfactory explanation for certain of the results on the changing $S$ tests and the changing $F_{0}$ tests. In the $S$ case, whereas P.G.G.'s results are roughly independent of $S$, L.A.D. shows an unusually low $V$ at $S=45 \mathrm{~mm}$ and A.Z.T. at $105 \mathrm{~mm}$. Similarly, in the $F_{0}$ case, whereas L.A.D.'s results are roughly independent of $F_{0}, A . Z$.T. shows a steady increase in $V$ with an increase in $F_{0}$, and P.G.G. shows a remarkably low $V$ at $F_{0}=2.5 \mathrm{~N}$. Obviously, these data on $V$ are much less orderly and much more variable across subjects than the data on the JND (\%).

Finally, it should be noted that the mean response bias $\bar{\beta}$ was relatively small in these experiments. More specifically, if one examines all the values of $\bar{\beta}$ corresponding to all of the points shown in Figures 2-5, one finds that in all cases $|\bar{\beta}| \leq 0.6$. Furthermore, if one excludes the data on changing base force $F_{0}$, one always has $|\bar{\beta}| \leq 0.09$. For the case of changing base force, the values of $\bar{\beta}$ were in the region $0.04 \leq \bar{\beta} \leq 0.59$.

\section{DISCUSSION}

According to the results shown in Figures 2-5, the force JND (\%) was relatively constant and was roughly $7 \%$ for the following sets of conditions:

$$
\begin{array}{ll}
2.5 \leq F_{0} \leq 10.0 \mathrm{~N} & D=20 \mathrm{~mm} \\
& S=105 \mathrm{~mm} \\
& V=\text { Most comfortable } \\
& \text { Mechanical wall } \\
& F_{0}=5 \mathrm{~N} \\
& D=10 \mathrm{~mm} \\
& V=\text { Most comfortable } \\
& \text { Mechanical wall } \\
& F_{0}=5 \mathrm{~N} \\
5 \leq D \leq 30 \mathrm{~mm} & S=105 \mathrm{~mm} \\
& V=\text { Most comfortable } \\
& \text { Mechanical wall } \\
& \text { Electrical cliff } \\
& F_{0}=5 \mathrm{~N} \\
& D=20 \mathrm{~mm} \\
& S=105 \mathrm{~mm} \\
& \text { Mechanical wall }
\end{array}
$$

Although not demonstrated in this set of experiments, the force JND (\%) must increase as (1) $F_{0}$ becomes very small, and (2) $D$ becomes very small. An increase in the Weber fraction as the reference becomes small holds for all sensory modalities and all variables (or, more precisely, all prothetic continua) and merely reflects the ex- 
istence of some irreducible internal noise in the system. The increase in this fraction as $D$ becomes small merely reflects the nature of the stimulus; if $D=0$ and there is no opportunity to sense the force, there is no way different forces can be discriminated. (Here $D=0$ refers to the situation in which there is neither kinesthetic nor cutaneous displacement.)

It should also be noted that the invariance of the Weber fraction with $D$ over the range of $D$ tested suggests that there is essentially no sensory or cognitive integration over the squeeze in this region of $D$. In other words, the interval over which discrimination improves as a result of integration (at whatever level in the system the integration takes place) must be less than or equal to $5 \mathrm{~mm}$. Unfortunately, the regions of small $F_{\mathrm{o}}$ and small $D$ in which the Weber fractions must be substantially larger than those shown could not be explored in our experiments, because of experiment limitations. (We plan in the future to build new equipment that will permit such measurements.)

Some further points that need to be stressed relate to the use of active finger motions in these tests. This feature of these tests implies that the stimulus on each trial can be regarded not only as a force stimulus but also as a work stimulus. Furthermore, since the force-versusdisplacement profiles were always constant (so that the work $W$ is given simply by the product $F \times D$ ), and since $D$ was always held fixed within a given run, there is no way of determining from these data whether the relevant underlying variable to which the subjects responded was force or work. In addition, and largely independently of this first question, the use of active finger motion in these tests enables the subject to obtain stimulus information through the kinesthetic/proprioceptive sensory system as well as the cutaneous sensory system. The issue of how much information is obtained through each of these sensory channels, a general issue that has a long and controversial history (e.g., see the discussion in Clark \& Horch, 1986), also cannot be addressed with the current data. The usual way of addressing this issue is to examine how performance is degraded when one or the other type of information is eliminated-for example, by the use of appropriate anesthetics or by employing paradigms in which the finger-plate presses against a passive supported finger, thus eliminating all noncutaneous information (force as well as work).

Finally, it should be noted that our results are at least roughly consistent with the results of previous, closely related experiments (e.g., see the review by Jones, 1989, as well as the review by Clark \& Horch, 1986). In most of these experiments, the task consisted of distinguishing among different weights. In Weber's classical work on weight estimation (see the translation by Ross \& Murray, 1978), differential thresholds for a 32-oz weight (corresponding to roughly $9 \mathrm{~N}$ ) on the hand were estimated by two methods, both of which compared the 32-oz reference in one hand to a lighter weight in the other hand. In one case, the hands lay motionless on a table (so that discrimination was based on touch alone); in the other case, the hands lifted the weights (so that muscles were also in- volved). For the 10-20 subjects tested, the JNDs (\%) were in the interval $17 \% \leq \mathrm{JND} \leq 50 \%$ (average $\approx 35 \%$ ) for the first method and $6 \% \leq \mathrm{JND} \leq 25 \%$ (average $\approx 10 \%$ ) for the second. In further tests on 4 subjects with a 32 -drachm (= $4 \mathrm{oz}$, corresponding roughly to $1 \mathrm{~N}$ ) reference, the results were essentially the same: $19 \% \leq \mathrm{JND} \leq 47 \%$ (average $\approx 29 \%$ ) for the first method, and $5 \% \leq \mathrm{JND} \leq 19 \%$ (average $\approx 10 \%$ ) for the second method. In other words, decreasing the reference weight by a factor of $8 \mathrm{had}$ essentially no effect. Given the differences between Weber's experimental procedures and ours (e.g., the involvement of more central muscle systems and the comparison across hands in Weber's tests), the results obtained by Weber for the active case are remarkably consistent with our results. Weber's experiments also suggest that the kinesthetic/ proprioceptive channel is considerably more sensitive than the cutaneous channel in this task.

More recent results on weight and mass discrimination are available in Brodie and Ross (1984), Raj, Ingty, and Devanandan (1985), and Ross and Brodie (1987) (see also the review by Jones, 1986). In the first study, differential thresholds were measured for roughly $2-0 z$ weights placed in the hand under four conditions (all of which involved successive comparisons in a single hand): (1) normal active lifting, (2) reflex active lifting (the reflex lift was induced by application of a vibrator to the tendon and lower part of the biceps muscle), (3) static raised (hand maintained at a fixed position above a horizontal surface), and (4) static supported (hand supported by resting forearm on a horizontal surface). The average Weber fractions for these four conditions were roughly $10 \%, 13 \%, 12 \%$, and $13 \%$. These results are consistent with those of Weber, in that the JND (\%) for the normal lifting mode is again roughly $10 \%$ and the JND (\%) for the static supported mode is significantly greater (although not nearly as large as that obtained by Weber for this mode). In the second study (Raj et al., 1985), weights in the range 0.7-18 oz (20-500 g) were applied to the middle phalanx of the middle finger of the right hand of both normal subjects and subjects with leprous neuropathy, and differential thresholds were measured for three conditions: (1) passive: (2) actively flexing the metacarpophalangeal joint, anc (3) actively flexing the elbow joint. The average results for these conditions (normal subjects) showed substan. tial increases in the JND (\%) for the lighter weights anc roughly equivalent degradations in sensitivity for Condi. tions 1 and 3 relative to Condition 2. Thus, for example the JND (\%) increased by more than a factor of 2 as thi reference weight decreased from roughly $3 \mathrm{oz}$ to $0.7 \mathrm{oz}$ and the JND (\%) for active flexing of the finger (Condi tion 2) was in many cases less than two thirds of that fo the active flexing of the elbow (Condition 3 ) or for th passive case (Condition 1). At $18 \mathrm{oz}$, the JNDs (\%) wer $10 \%, 14 \%$, and $19 \%$ for Conditions 2,3 , and 1 , respec tively; at $3 \mathrm{oz}$, they were $13 \%, 33 \%$, and $28 \%$, respec tively; and at $0.7 \mathrm{oz}$, they were $28 \%, 84 \%$, and $89 \%$ respectively. These results again appear to be roughly con sistent with the other data reported. In the third stud. (Ross \& Brodie, 1987), cylindrical weights in the rang 
1.8-18 oz (50-500 g) were sensed under three conditions: (1) weight-grasping the cylinder firmly and moving it up and down; (2) mass-firm-grasping the cylinder firmly and swinging it from side to side; and (3) mass-looseholding the cylinder loosely and shaking it from side to side. The results indicate JNDs (\%) in the range $8 \%-14 \%$, with the best results obtained for the weight condition and the larger weights.

Further relevant results have recently been obtained by Jones (in press) from experiments in which the subject was required to match the force exerted by the elbow flexor muscles of one arm (isometric contraction) with an equal force generated by the contralateral limb. Using the standard deviation of the observed matching force at a fixed reference force as the measure of the differential threshold for that reference force, Jones obtained JNDs (\%) in the range $5 \%-9 \%$ (mean $7 \%$ ) across reference forces that varied from $15 \%$ to $85 \%$ of the maximum voluntary contraction force (which was a few hundred Newtons). Although previous results (e.g., see the discussion of past work in Jones, 1986) suggest that the difference in JNDs (\%) associated with the difference between isometric contraction and active motion might be small, the similarity between these data and our own is remarkable.

In general, it appears that the force JND (\%) is approximately $7 \%$ over a wide range of conditions involving great variation in force magnitude, muscle system, and experimental method, provided only that the kinesthetic/ proprioceptive senses (and not only the cutaneous sense) are involved.

In future work, we plan to extend the basic results on manual force JNDs reported in this paper to include not only cases in which kinesthetic/proprioceptive information is eliminated, but also cases in which the magnitudes of the forces and displacements are greatly reduced, as well as cases in which there are both a " $\mathrm{dc}$ " and an "ac" (masking) force component (with the resolution for the dc component measured as a function of the strength of the ac component). In addition, we intend to pursue the question of whether it is force or work that is sensed in the active case. Finally, we intend to extend our work to include discrimination of compliance (the slope $K_{1}$ in the equation $F=K_{1} X$ ), viscosity (the slope $K_{2}$ in the equation $F=K_{2} \mathrm{~d} X / \mathrm{d} t$ ), mass (the slope $K_{3}$ in $F=K_{3} \mathrm{~d}^{2} X / \mathrm{d} t^{2}$ ), and other higher order terms in the dependence of force $F$ on displacement $X$ and its time derivatives.

\section{REFERENCES}

Berliner, J. E., \& Durlach, N. I. (1973). Intensity perception: IV. Resolution in roving-level discrimination. Journal of the Acoustical Society of America, 53, 1270-1287.

Brodie, E. E., \& Ross, H. E. (1984). Sensorimotor mechanisms in weight discrimination. Perception \& Psychophysics, 36, 477-481.

Clark, F. J., \& HoRCH, K. W. (1986). Kinesthesia. In L. R. Boff, L. Kaufman, \& J. P. Thomas (Eds.), Handbook of perception and human performance (Vol. 1, chap. 13). New York: Wiley.

Durlach, N. I., Delhorne, L. A., Wong, A., Ko, W. Y., Rabinowitz, W. M., \& Hollerbach, J. (1989). Manual discrimi- nation and identification of length by the finger-span method. Perception \& Psychophysics, 46, 29-38.

Jones, L. A. (1986). Perception of force and weight: Theory and research. Psychological Bulletin, 100, 29-42.

JONEs, L. A. (1989). The assessment of hand function: A critical review of techniques. Journal of Hand Surgery, 14A, 221-228.

JoNEs, L. A. (in press). Matching forces: Constant errors and differential thresholds. Perception.

Ras, D. V., Ingty, K., \&evanandan, M. S. (1985). Weight appreciation in the hand in normal subjects and in patients with leprous neuropathy. Brain, 108, 95-102.

Ross, H. E., \& Brodie, E. E. (1987). Weber fractions for weight and mass as a function of stimulus intensity. Quarterly Journal of Ex perimental Psychology, 39A, 77-88.

Ross, H. E., MURRay, D. J. (1978). E. H. Weber: The sense of touch. New York: Academic Press.

\section{APPENDIX A}

A system block diagram for the apparatus pictured in Figure 1 is shown in Figure A1. A dc linear-motion motor served as a back-drivable actuator for the movable finger plate. Control was provided by a TMS320C20 Digital Signal Processing board hosted by an IBM-compatible personal computer. Force, displacement, and velocity information from sensors attached to the movable finger plate were fed back to the controller through a three-channel, 16-bit $\mathrm{A} / \mathrm{D}$ converter at $2.5-\mathrm{kHz}$ sampling rate. The force sensor was a BLH semiconductor strain gage, the position sensor was a noncontacting FLDT by Sunpower, and the velocity sensor was an inductive type from Transducer Systems. In order to eliminate errors in the measurement of the force applied to the movable plate by the thumb, a cylindrical roller mounted on the movable plate served as a contact point for the thumb (see Figure 1). This not only ensured that the force was always applied at a constant height above the location of the force sensor in the plate (so that the effective lever arm length remained constant), but also that the force was always applied in the direction perpendicular to the plate in the vertical plane.

\section{SYSTEM BLOCK DIAGRAM}

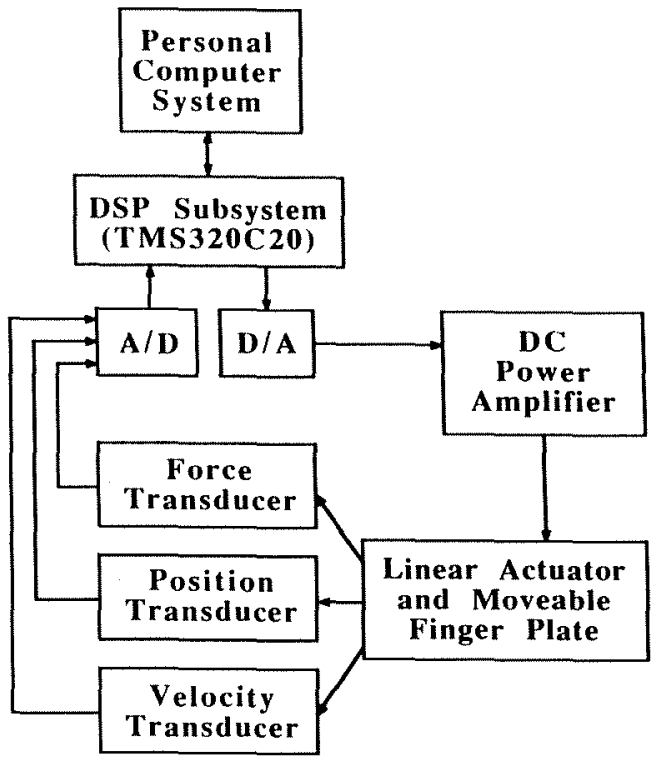

Figure A1. System diagram of the experimental apparatus. 


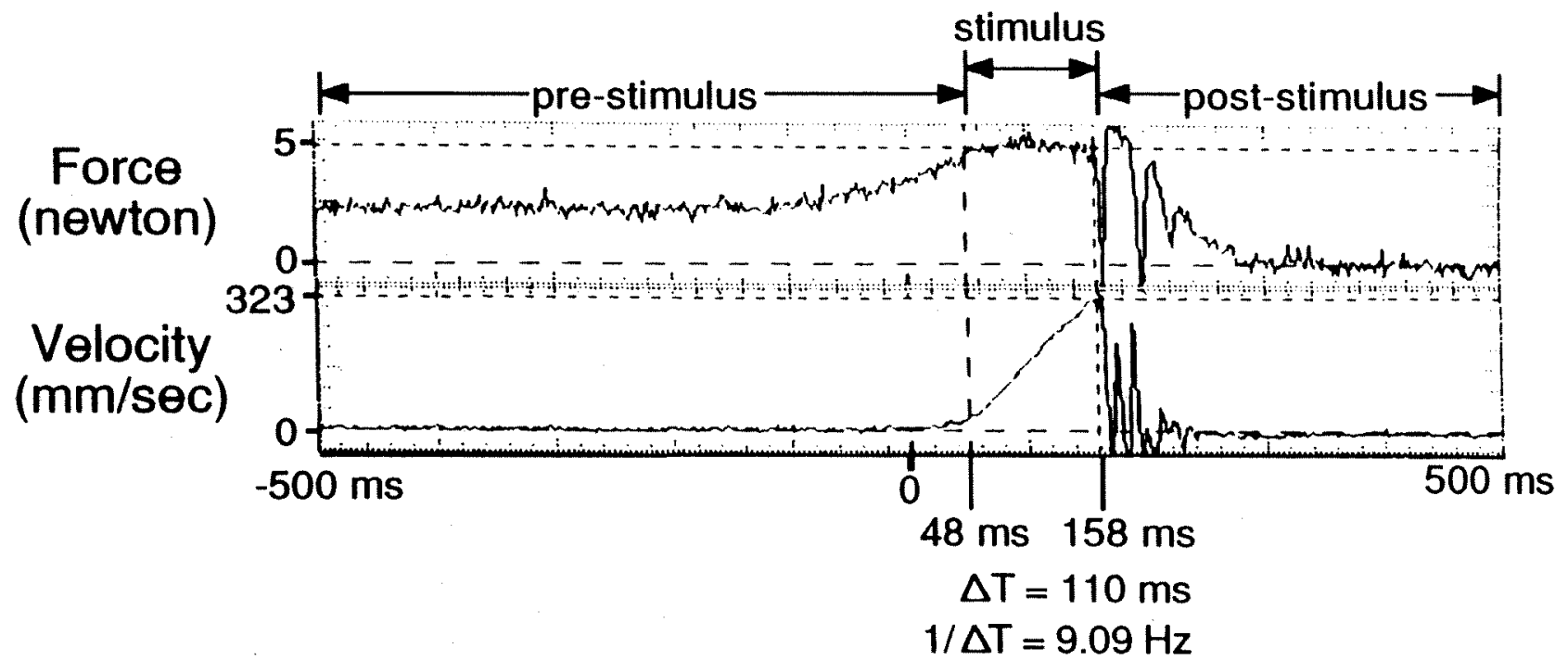

Figure A2. Recording of force and velocity of the finger plate during a push. The two horizontal dashed lines with the upper trace indicate 0 and $5 \mathrm{~N}$ of force, respectively. The two horizontal dashed lines with the lower trace indicate $0 \mathrm{and} 323 \mathrm{~mm} / \mathrm{sec}$ of pushing velocity, respectively. The two vertical dashed lines indicate the beginning and ending of the finger-plate displacement ( $20 \mathrm{~mm})$, respectively. The average velocity and acceleration were $182 \mathrm{~mm} / \mathrm{sec}$ and $2,936 \mathrm{~mm} / \mathrm{sec}^{2}(\approx 1 / 3 \mathrm{~g})$, which were larger than those involved in data collection (see Figure 5 and Table 2 ). The push was terminated by a mechanical wall.

The control signal was delivered to the actuator through a 16bit D/A converter and a Techron de power amplifier, which acted as a voltage-controlled current source. Except for the motor, all components in the control loop had a wide bandwidth and linearity range. The force/current gain of the motor was approximately constant over a stroke range of $55 \mathrm{~mm}$. Within this stroke range, the motor could be modeled as a second-order system up to $200 \mathrm{~Hz}$. The motor system function had a constant magnitude up to $20 \mathrm{~Hz}$ and a strong resonance at around $50 \mathrm{~Hz}$. The friction force of the motor-finger interface assembly was both position and direction dependent, but always less than $1 \mathrm{~N}$. The cascaded noise level from the force sensor and the A/D converter was less than $0.05 \mathrm{~N}$.

Force control was accomplished with acceleration compensation. In other words, when the subject pushed the finger plate with an acceleration, the acceleration component in the total force felt by the subject was measured and subtracted from the total force so that the force presented to the subject remained constant, independently of how fast the subject pushed, up to the effective closed-loop bandwidth of the system $(>10 \mathrm{~Hz})$. Figure A2 shows that when the finger interface was pushed at speeds faster than those employed in this study by the subjects, the system was able to keep the force on the thumb constant. The range of constant force was $2-30 \mathrm{~N}$, with a resolution of $0.05 \mathrm{~N}$. The difference between the specified force and the actual steady-state force was less than $3 \%$.

\section{APPENDIX B}

\section{ROC Curves}

The manner in which we have computed sensitivity index $d^{\prime}$ and bias $\beta$ in this paper assumes that the underlying density functions associated with the two stimuli being discriminated are normal and of equal variance. In order to determine the validity of this assumption, we measured receiver operating characteristics (ROCs) for the 3 subjects G.Y.O., L.A.D., and P.G.G. for the case in which $F_{0}=5 \mathrm{~N}, \Delta F / F_{0}=10 \%, D=20 \mathrm{~mm}$,
$S=105 \mathrm{~mm}$, and the termination method was MW. ROCs were determined by varying the a priori probabilities of the stimuli $F_{0}$ and $F_{0}+\Delta F$ and informing the subject of these probabilities before each run (so that the response criterion would be altered accordingly). The resulting data, plotted on normal-deviate coordinates (and representing roughly 6,000 trials for each of the 3 subjects), are shown in Figure B1. With these coordinates,

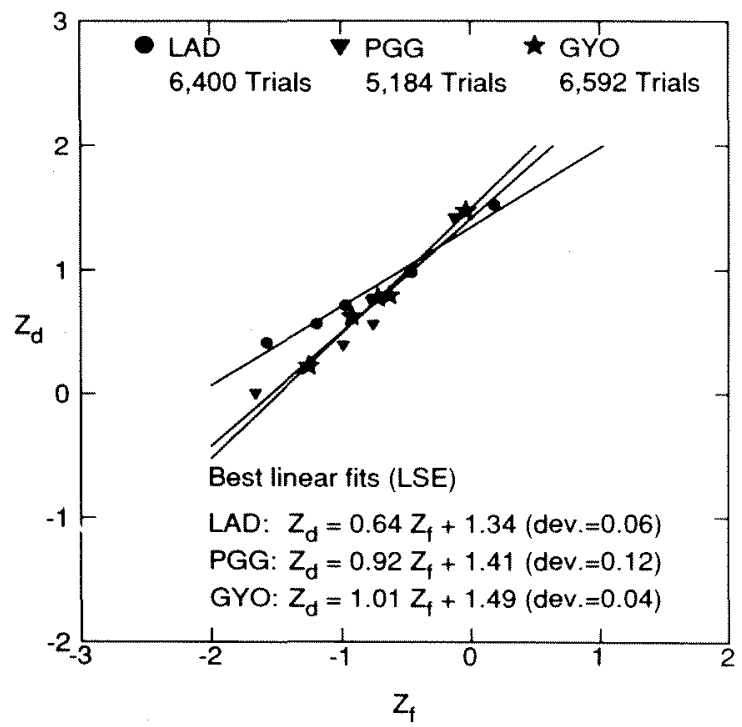

Figure B1. Receiver operating characteristics in normal-deviate coordinates. The a priori probabilities for presenting the reference force were $1 / 10,3 / 10,5 / 10,7 / 10$, and $9 / 10$. Reference force $F_{0}$ was $5 \mathrm{~N}$, force increment was $10 \%$, pushing distance $D$ was $20 \mathrm{~mm}$, initial finger-span $S$ was $105 \mathrm{~mm}$, and termination was by mechanical wall (MW). Average slope is 0.86 ; intercept, 1.42 . The numbers in parentheses represent root mean square deviations of the straightline fits. 
Table B1

Ratio of Standard Deviations of the Measured $d$ 's to That of the Simulated $d$ 's

\begin{tabular}{lccccc}
\hline Subject & $\begin{array}{c}\Delta F / F_{0} \\
=5 \%\end{array}$ & $\begin{array}{c}\Delta F / F_{0} \\
=10 \%\end{array}$ & $\begin{array}{c}\Delta F / F_{0} \\
=15 \%\end{array}$ & $\begin{array}{c}\Delta F / F_{0} \\
=20 \%\end{array}$ & Average \\
\hline L.A.D. & 1.16 & 1.22 & 1.26 & 1.22 & 1.22 \\
P.G.G. & 1.03 & 1.05 & 1.27 & 1.10 & 1.11 \\
A.Z.T. & 0.84 & 1.20 & 1.26 & 1.24 & 1.14 \\
Average & 1.01 & 1.16 & 1.26 & 1.19 & 1.16 \\
\hline
\end{tabular}

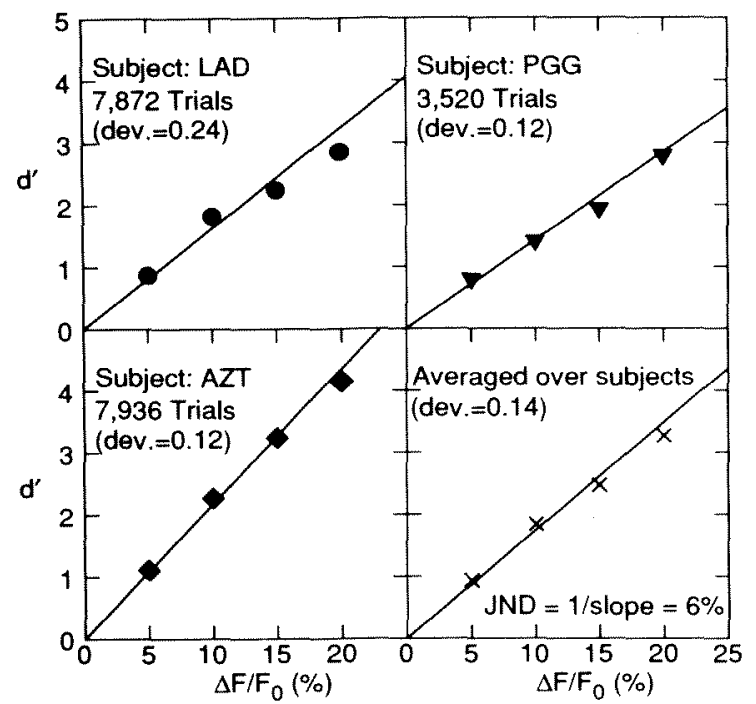

Figure B2. Sensitivity index $d^{\prime}$ versus percentage force increment $\Delta F / F_{0}$. The slope of each solid line is an average of the slopes of each datum point. Reference force $F_{0}$ was $5 \mathrm{~N}$, pushing distance $D$ was $20 \mathrm{~mm}$, initial finger-span $S$ was $105 \mathrm{~mm}$, and termination was by mechanical wall (MW). The numbers in parentheses represent root mean square deviations of the straight-line fits. the data are consistent with the assumptions to the extent that they lie on a straight line of slope unity. According to these results, the points are well fit by straight lines (the root mean square deviations of the points from the least-square-error fit of straight lines are $0.06,0.12$, and 0.04 for Subjects L.A.D., P.G.G., and G.Y.O., respectively). Also, the slopes of these straight lines are reasonably close to unity $(0.92$ and 1.01$)$ for 2 of the 3 subjects (P.G.G. and G.Y.O.). The substantially smaller slope, 0.64, for Subject L.A.D. is puzzling. However, the resulting distortion in the estimates of $d^{\prime}$ for L.A.D. does not appear to be large, relative to the fluctuations among the data points shown in Figures 2-5 or to influence any of the conclusions stated in the discussion of these data.

\section{Variability of $d^{\prime}$ Estimates}

In order to evaluate our assumption that the trials are independent and constitute a Bernoulli process with time-invariant statistic characteristics, we compared the variance of our empirical $d^{\prime}$ measurements (based on 64-trial runs) with the variance derived from an appropriate computer simulation of a Bernoulli process. Our estimates of the variance for the empirical case were based on the results (totaling over 400 64-trial runs) obtained by Subjects A.Z.T., L.A.D., and P.G.G. for the standard parameter values $F_{0}=5 \mathrm{~N}, S=105 \mathrm{~mm}, D=20 \mathrm{~mm}$, termination method $=\mathrm{MW}$, and $\Delta F / F_{0}=5 \%, 10 \%, 15 \%$, and $20 \%$. Our estimates for the simulation were based on 10,000 runs so that stabilized results were guaranteed. The ratio of the experimental standard deviation to the simulated standard deviation, averaged over the 4 values of $\Delta F / F_{0}$ and the 3 subjects, was 1.16. The values of the ratio for the individual subjects (averaged over $\Delta F / F_{0}$ ) were $1.22,1.11$, and 1.14 for L.A.D., P.G.G., and A.Z.T., respectively. The values for each $\Delta F / F_{0}$ (averaged over the subjects) were $1.01,1.16,1.26$, and 1.19 for $5 \%, 10 \%, 15 \%$, and $20 \%$, respectively. The 12 individual values of the ratio are shown in Table B1. In view of the long time period over which these experiments were performed (a number of months) and the many associated opportunities for

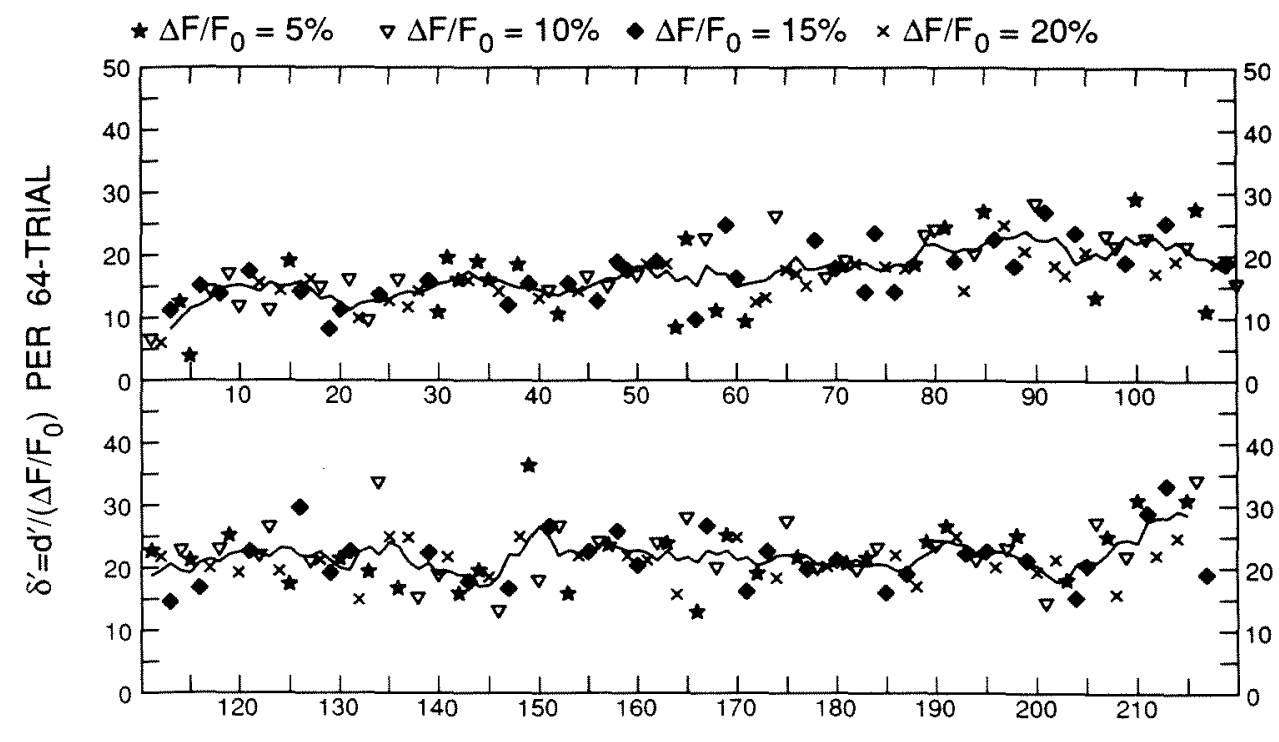

RUN NUMBER M

Figure C1. Training effects for Subject A.Z.T. with standard parameter values. Reference force $F_{0}$ was $5 \mathrm{~N}$, pushing distance $D$ was $20 \mathrm{~mm}$, initial finger-span $S$ was $105 \mathrm{~mm}$, and termination was by mechanical wall (MW). The solid line is a 5-point moving average of the data. Symbol code is specified on the graph. 


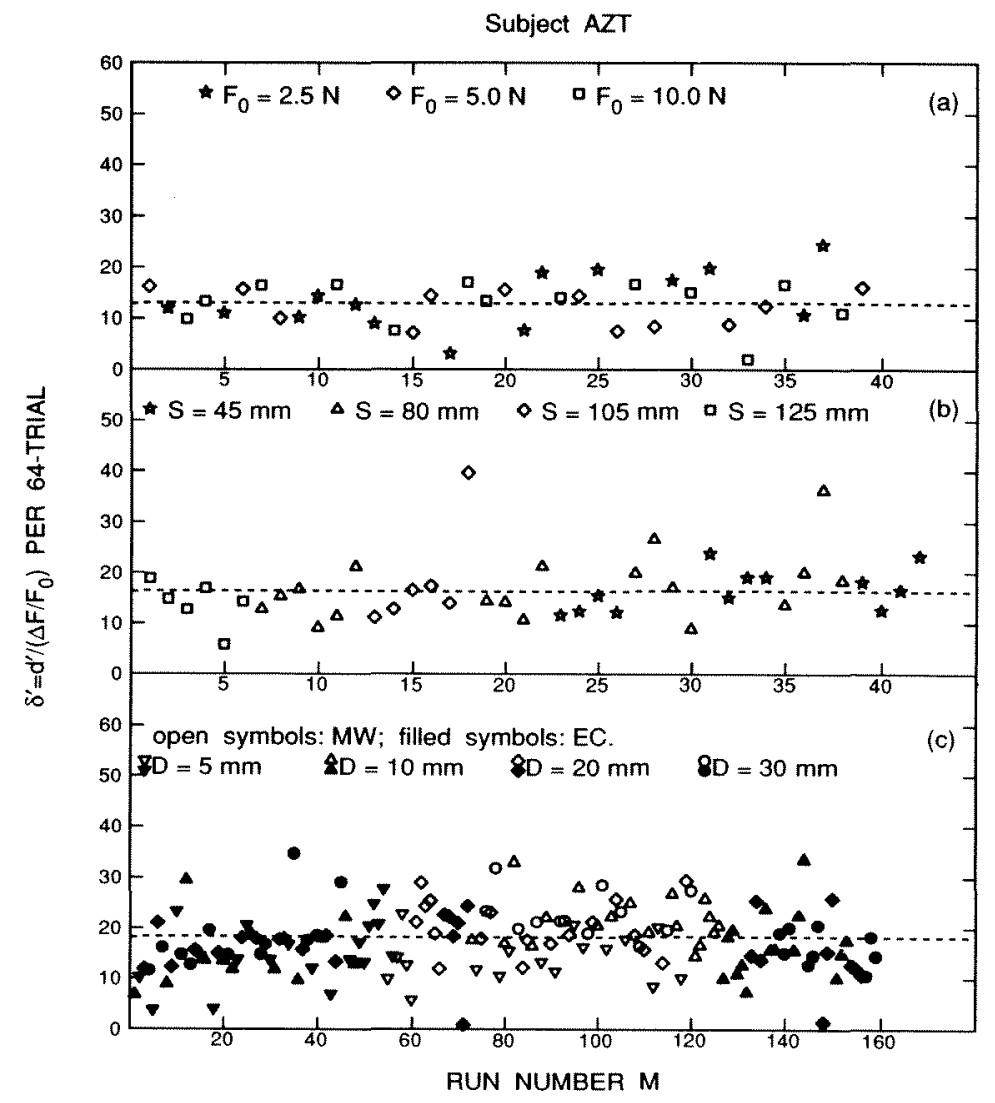

Figure C2. Training effects for the tests in which (a) $F_{0}$, (b) $S$, or (c) $D$ was changed for Subject A.Z.T. (see Table 1 for parameter settings). Dashed lines indicate average performance level corresponding to JNDs shown in Figures 2-4. Open symbols show results for mechanical wall (MW), filled symbols for electrical cliff (EC). Note the relatively poor results for the first few $\mathrm{EC}$ runs in Graph c.

nonstationarity to occur, we regard these results as supportive of our assumptions.

\section{Proportionality of $d^{\prime}$ and $\Delta F$}

A further assumption of our data processing is that $d^{\prime}$ is proportional to $\Delta F$. In Figure $B 2$, we have plotted the dependence of $d^{\prime}$ on $\Delta F / F_{0}$ measured for the standard parameter setting, together with average straight-line fits (constrained to go through the origin and to have a slope that is the average of the slopes determined by the individual points). The root mean square deviation of the points from the lines is $0.24,0.12$, and 0.12 for Subjects L.A.D., P.G.G., and A.Z.T., respectively.

\section{APPENDIX C}

The effects of training were examined by plotting the quantity $\delta^{\prime}=d^{\prime} /\left(\Delta F / F_{0}\right)$ as a function of run number $M$. In only two cases was a substantial training effect apparent: (1) when the subject first began participating in the series of experiments, and (2) when the method of terminating the push was changed from MW to EC. In all other cases, the training effect was negligible.

Figure $\mathrm{C} 1$ shows the training effect that occurred in the tests with the standard parameter setting at the beginning of our experiments for Subject A.Z.T. Different symbols are used to code different values of $\Delta F$. The smoothed (moving-average) version of $\delta^{\prime}$ appears to have an approximately linear rising phase followed, at about $M=80$, by a flat "saturation" phase.

Figure C2 shows (for Subject A.Z.T.) the lack of additional training effects for the tests in which $F_{0}, S$, or $D$ was changed but MW termination was maintained, and then the return of training effects when the EC termination procedure was introduced into the tests of changing $D$. In this figure, the different values of $\Delta F$ are ignored and different symbols are used to code the different values of $F_{0}, S$, and $D$. When one examines the training effects for all subjects and all conditions, one finds that, on the average, the performance level $\delta^{\prime}$ was increased by over $50 \%$ after training with the MW stimulus termination and by over $100 \%$ after training with EC. All subjects reported a different "feel" when the mode of stimulus termination changed; and EC seemed more difficult initially. The number of runs required to achieve stable performance, $M_{s}$, varied from subject to subject, indicating that each subject learned the tasks at a different pace. The average $M_{s}$ for both modes of stimulus termination was about 45 runs. Results reported in the body of this paper are based on runs performed after stable performance was achieved.

(Manuscript received July 19, 1990; revision accepted for publication January 17, 1991.) 